\title{
GIS 技术和数字化测绘技术在工程测量中的应用
}

\author{
骆采采 1 周剑虹 2 \\ 1 杭州呈元测绘有限公司 2 巨化集团公司 \\ DOI:10.32629/gmsm.v2i6.392
}

[摘 要] 随着我国市场经济的飞速发展, 我国也逐渐的开始进行一些高质量的工程项目的建设。基于GIS技术和数字化测绘技术在工程测量工 作中所起到的重要作用, 我国政府和相关部门也越来越重视GIS技术和数字化测绘技术在工程测量中的应用。基于此,下文就我国工程测量的概 述、GIS技术和数字化测绘技术的介绍和在我国工程测量中的应用等方面做了简单分析,希望对我国工程项目的发展有所启示和帮助。

[关键词] GIS技术; 数字化测绘技术; 工程测量；应用

\section{引言}

工程测量是我国工程项目建设的基础环节, 保证工程测量的质量, 对 于提高工程项目的整体质量、促进我国经济的发展等方面都有着十分重要 的现实意义。 ${ }^{[1]}$ 因此, 我国相关的工程建设企业应该加强对工程测量技术 的分析和研究, 积极应用新时期下的GIS技术和数字化测绘技术, 明确GIS 技术和数字化测绘技术的优势, 实现其在工程测量中的良好应用, 保证工 程项目的质量, 促进企业的长久进步。

\section{1 工程测量的概述}

工程测量从概念上来讲指的是对建筑、水利、交通等工程项目的测量 工作。工程测量工作是工程企业对工程项目正式开始设计施工的基础工作, 工程测量的数据是工程项目施工的依据。因此, 工程测量数据的质量直接 影响着整个工程项目的施工质量。工程测量数据从内容上包括工程的图 形、模型、符号信息等, 在很大程度上可以提升工程项目的建设水平。

现阶段, 随着GIS技术和数字化测绘技术在我国工程测量中的广泛应 用, 在很大程度上提高了我国工程测量的准确度, 提高了工程的整体质量。 就目前我国工程测量的基本现状来看, GIS技术和数字化测绘技术在我国 工程测量中的应用已经较为成熟。

\section{GIS 技术与数字化测绘技术的简单介绍}

\subsection{GIS技术的的简单介绍}

GIS技术是我国科技信息技术飞速发展下的科技成果，同时也是新时 期下的一种先进的工程测量技术。通过在工程测量中应用GIS技术, 提高了 工程项目的计算机制图水平。因此, 近几年来了, GIS技术也逐渐的受到了 我国工程企业的重视。GIS技术在工程项目测量中的应用优势主要体现在 以下几个方面: 第一, GIS技术是多种新型技术的融合, 因此, 在实际的工程 测量应用中, 在很大程度上可以实现工程测量的各种图像图形信息的高度 集成,有利于提高工程信息的有效性,并提高工程测量信息的精确度; 第 二, GIS技术在应用过程中, 可以升级地理信息系统, 实现工程测量信息的 信息共享, 有利于企业对不同信息进行统筹管理; 第三, GIS技术在发展完 善的过程中还结合了新时期下的3D技术和虚拟技术, 进而在功能上更加全 面, 大大提高了工程项目的测量水平。

2. 2 数字化测绘技术的简单介绍

2. 2. 1 数字化测绘技术的概念

数字化测绘技术也是我国新时期下的一种新型技术。数字化测绘技术 从概念上来讲指的是以处理数字信息为核心的一种信息系统,在我国各行 各业都有着十分重要的应用价值。数字化测绘技术的技术类型有很多, 其 中, 最主要的类型是通讯技术类型。因此, 数字化测绘技术也具有很大的应 用优势, 值得进行更大范围的推广和应用 ${ }^{[2]}$ 。

2.2.2数字化测绘技术的优势特点
数字化测绘技术和我国传统的测绘技术相比较, 具有很多的优势, 在 很大程度上也满足了新时期下工程企业工程测量的需求。具体体现在以下 几个方面: 第一, 通过应用数字化测绘技术, 有利于工程企业实现工程数据 的自动化处理。数字化测绘技术中的计算机技术可以对工程数据进行自动 绘图, 同时, 自动绘图的质量也非常高; 第二, 通过应用数字化测绘技术, 可以帮助工程企业对工程绘图进行相关的编辑处理。因此我国工程项目在 实际的建设过程中, 经常会遇到临时修改工程绘图的情况, 应用传统的绘 图技术进行改动通常情况下很难保证图形的完整性, 但是, 应用数字化测 绘技术, 企业可以在不改变图形整体的基础上对需要修改的图形直接进行 改动, 在很大程度上保证了企业工程建设质量; 第三, 合理应用数字化测绘 技术中的TRK技术, 有利于企业提高测量数据的准确性, 为工程的数据测绘 打下坚实的基础。

\section{GIS 技术在工程测量中的具体应用}

3. 1 在数据获取方面的应用

GIS技术在工程测量中的最主要的应用就是应用于工程项目的数据获 取方面。工程测量最重要的环境就是获取测量数据, 通过应用GIS技术, 相 关测量人员可以结合工程项目的图例说明, 使得工程测量数据更加直观和 形象。同时, 通过应用GIS技术, 测量人员还可以对测量数据进行标记, 获取 相关的工程电子地图。

\section{2 在数据查询方面的应用}

GIS技术应用于工程测量工作中的数据查询方面, 可以帮助企业迅速 的了解并掌握工程测量的全部信息, 进而完成数据的分析和判断, 实现工 程测量数据的检测。同时, GIS技术在实际的工程测量的应用过程中, 通常 情况下是通过文字和图片的形式进行数据表达的, 在一定程度上也使得工 程测量信息更加的形象化, 有利于测量人员进行迅速的篮选和查询, 提高 测量工作的效率。

\section{3 在描述空间分析结果方面的应用}

GIS技术还具有很强的空间分析能力, 在实际的工程测量应用过程中 GIS技术可以实现对测量数据的叠加分析、地形分析、缓冲区分析和网络 分析等各个方面的分析, 大大提高了工程测量数据的分析水平。同时, GIS 技术还可以获得模拟工程中的空间分析成果, 保证分析结果的有效性。最 直接的例子就是, 工程企业在进行工程测量时, 可以利用GIS技术实现对工 程项目测量的地理信息的描述和分析,进而, 保证工程项目的测量效果。另 外, GIS技术还有一个应用优势, 就是无论工程项目的地理条件是否发生变 化, GIS技术都可以对工程的缓冲区进行准确分析。

\section{4 数字化测绘技术在工程测量中的应用}

近几年来, 数字化测绘技术在我国工程项目的测量工作中的应用也越 来越广泛, 而且也取得了一定的应用成果。工程企业通过应用数字化测绘 


\title{
现代测绘技术在工程测量中的应用及完善策略
}

\author{
林青 陈川
}

扬州市勘测设计研究院有限公司

DOI:10.32629/gmsm.v2i6.440

[ 摘 要] 现代社会经济的不断发展对我国工程测量工作提出了更高的要求,在开展具体工作过程中,科学应用现代测绘技术能够对其工程质量 进行更高程度的保障,本文首先分析工程测量过程中现代测绘技术的具体应用,然后以此为基础,进一步探究其完善策略,希望能够对其工程测量 效果进行更高程度的保障。

[关键词] 现代测绘技术; 工程测量; 具体应用; 科学完善

\section{引言}

在开展工程建设过程中, 现代测绘技术的科学应用具有极其重要的现 实意义, 是其相关工作开展的重要保障, 必须对其加强重视, 为了进一步明 确在工程测量过程中如何更为科学的应用现代测绘技术, 特此展开本次研 究, 希望能够有效推进我国现代工程建设。

\section{1 工程测量中现代测绘技术应用}

1. 1 高程控制测量

在具体进行高程控制测量工作时, 相关工作人员首先需要基于测绘目 标在具体施工范围进行高程控制点的科学设置, 对其控制点位置进行合理 确定, 在具体设定高程控制点时, 需要确保相邻控制点处于相同水准路线, 以此为基础, 能够实现高程控制网的科学构建, 确保全面监控测绘区域, 在 具体工作过程中, 还需要对其以下几个方面加强重视。其一, 控制闭合水准 路线, 在进行高层控制网建设过程中, 需要科学设计等外闭合水准线, 同时, 严格基于相关规定方法进行科学控制, 以此为基础进行具体工作, 才能使 其高程控制网具有更高的精确度。其二, 在开展工程测量工作时, 测站计量 结果会对其整体测量造成很大程度的影响, 基于此, 相关工作人员需要对 其测站计量进行严格有效的检测, 确保其精确度, 科学应用相关计量方法 和公式进行精确核算, 对其计量数据准确性进行更高程度的保障。其三科 学计算高差, 在具体计算高度差时, 现场工作人员需要严格基于相关规章 制度进行反复核算, 确保高差数值具有更高的精确性, 随后才可以将其用 于测绘计量工作, 为其后期数据计量工作提供必要的数据支持。其四, 进行 闭合度测量, 该项工作的主要目的在于确保实际闭合差高度符合标准数值 间, 如果发现二者之间存在差错, 必须对其差错原因进行综合分析, 并以此 为基础进行科学改正。一般而言, 如果其闭合差值出现问题, 相关工作人员 必须重新测量闭合度, 为了确保相关数据具有更高的准确性, 测绘人员需 要尽量进行多次测量, 然后通过对比分析出现闭合差的原因, 进而确保测 量具有更高的精确度。下图1为在三角高程测量中全站仪的应用。

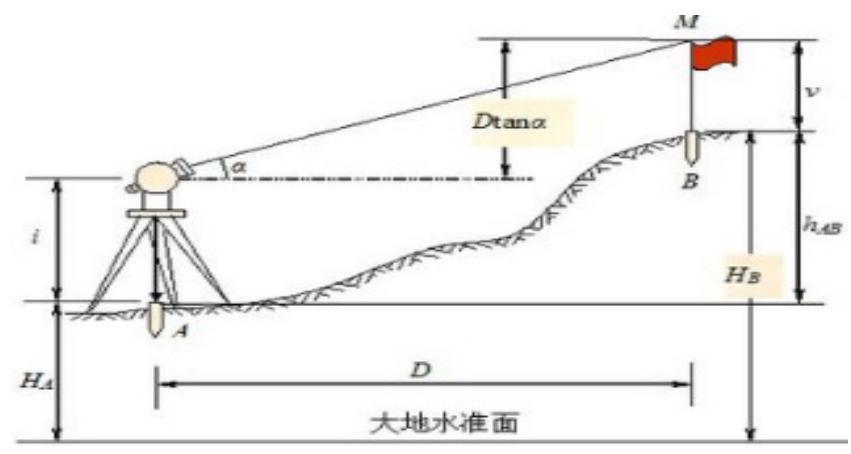

图1 高程测量测量方法

1.2 平面控制测量

在进行工程测量过程中, 平面测绘是其不可或缺的重要环节, 其结果 会在很大程度内影响建筑质量和施工质量, 进而在很大程度内影响项目建 设经济效益。基于此, 必须高度重视平面空间测量, 确保测量结果具有更高 的精确性, 为顺利开展工程施工提供必要的数据保障。与此同时, 在具体实 施平面控制测量过程中, 需要在其具体工作范围进行测量控制网的科学建 设。如果想要确保进行平面测量控制网的科学构建, 首先需要选择测量控 制点, 设计人员在具体选择测量控制点, 必须对其项目具体情况进行综合 考虑, 利用现代测绘技术科学选择控制点, 避免盲目选择, 在控制点选择完 成之后。相关工作人员需要在控制点上安装测量标志, 然后有效连接控制 点, 实现平面控制网的有效形成。基于平面控制网在测绘区域科学实施测 绘工作 ${ }^{[1]}$ 。在此过程中, 安装平面测量网的具体位置对其测绘数据具有一 定程度的决定性影响, 因此, 相关工作人员基于相关规章制度进行控制点 选择和连接。在具体开展工程测量工作时, 平面控制测量试题极为重要的 一项内容, 为了确保平面控制网具有更高的测绘质量, 现场工作人员在具 体进行工程测量科学应用GPS技术进行测量控制点的科学选择, 确保测量
技术, 可以有效提高工程项目的建设速度, 保证工程质量。目前, 数字化测 绘技术的发展目标是实现工程测绘的数字化和自动化, 但是, 与此同时, 数 字化测绘技术也不应该抛弃我国传统的工程测绘技术。在实际的应用过程 中, 工程企业应该注意在传统测绘技术的基础上有效融合数字化的测绘技 术, 进而保证测绘效果, 弥补我国传统测绘技术上的不足, 进而保证工程测 量的工作效果, 促进我国测绘技术的发展 ${ }^{[3]}$ 。

\section{5 结束语}

综上所述, 随着我国科学技术的发展进步, 我国的工程企业在工程测 量工作中也逐渐的开始重视新时期下的GIS技术和数字化测绘技术的分析 和研究, 进而明确GIS技术和数字化测绘技术的特点和优势, 在传统测量技
术的基础上积极引进新时期下的新型技术, 实现GIS技术和数字化测绘技 术在工程测量数据获取、查询和分析等方面的良好应用, 提高工程测量结 果的准确性, 保证工程项目的建设质量, 为我国工程项目的发展保驾护航。

[参考文献]

[1]韦文.GIS技术和数字化测绘技术在工程测量中的应用 [J]. 电子技 术与软件工程,2019,(14):68-69.

[2]胡奇秀.试论数字化测绘技术在水利工程测量中的应用 [J].建材与 装饰,2019,(18):207-208.

[3]雷时光.浅析数字化测绘技术在工程测量中的运用[J].中国管理信 息化,2019,22(12):131-132. 